AZEVEDO AM; ANDRADE JÚNIOR VC; PEDROSA CE; OLIVEIRA CM; DORNAS MFS; VALADARES NR. 2015. Agrupamento multivariado de curvas na seleção de cultivares de alface quanto à conservação pós-colheita. Horticultura Brasileira 33: 362-367. DOI: http://dx.doi.org/10.1590/S0102053620150000300014

\title{
Agrupamento multivariado de curvas na seleção de cultivares de alface quanto à conservação pós-colheita
}

\author{
Alcinei M Azevedo; Valter C Andrade Júnior²; Carlos E Pedrosa²; Celso M Oliveira²; Marcus FS \\ Dornas $^{2}$; Nermy R Valadares ${ }^{2}$ \\ 'Universidade Federal de Viçosa, UFV, Viçosa-MG, Brasil; alcineimistico@hotmail.com; ${ }^{2}$ Universidade Federal dos Vales do \\ Jequitinhonha e Mucuri, UFVJM, Diamantina-MG, Brasil; valterjr@ufvjm.edu.br; carlosenrrik@yahoo.com.br; celsodoliveira@yahoo. \\ com.br;mfsdornas@yahoo.com.br; nermyvaladares@hotmail.com
}

\begin{abstract}
RESUMO
Um dos maiores problemas para a comercialização da alface é a sua curta vida de prateleira devido à perda de qualidade. Uma forma de estudar o declínio da qualidade dos vegetais em função do tempo após a colheita é o método gráfico, porém esta estratégia pode ser dificultada caso seja avaliado um grande número de tratamentos. Neste contexto, o agrupamento de curvas utilizando a análise multivariada é uma alternativa viável. Objetivou-se neste trabalho selecionar cultivares de alface quanto à conservação pós-colheita por meio do agrupamento multivariado de curvas. Foram avaliadas quinze cultivares de alface quanto à perda acumulada de matéria fresca (PAMF) e grau de descoloração (GD) ao longo de 72 horas após a colheita. Foram ajustados 13 modelos de regressão, verificando melhor ajuste do modelo linear simples para a PAMF e do modelo logístico para o GD. Para ambas as características avaliadas agruparam-se as cultivares em seis grupos. As cultivares 'Romana Balão' e 'Vitória de Santo Antão' são as mais resistentes quanto à desidratação e descoloração no armazenamento em temperatura ambiente, sendo indicadas para a produção, comercialização e como genitores em programas de melhoramento.
\end{abstract}

Palavras-chave: Lactuca sativa, modelo linear, modelo não-linear, vida de prateleira, estatística multivariada.

\begin{abstract}
Multivariate grouping of curves in the study of post-harvest conservation of lettuce cultivars

One of the main problems for the commercialization of lettuce is the short shelf life due to loss of quality. One way to study the decline of plant quality in function of time after harvest is the graphical method, however this strategy may become difficult if a great number of treatments is evaluated. So, the grouping of curves using multivariate analysis can be a viable alternative. The objective of this work was to select lettuce cultivars presenting better postharvest conservation through multivariate clustering curves. Fifteen lettuce cultivars were evaluated in terms of cumulative loss of fresh matter (PAMF) and discoloration degree (GD) during 72 hours after harvest. Thirteen regression models were adjusted, checking the best fit of the first-degree polynomial model for the PAMF and the logistic model for the GD. For both traits, the cultivars were grouped into six groups. The cultivars 'Romana Balão' and 'Vitória de Santo Antão' are the most resistant to dehydration and discoloration in storage at environmental temperature, being recommended to production, marketing and as parents in breeding programs.
\end{abstract}

Keywords: Lactuca sativa, linear model, nonlinear model, shelf life, multivariate statistics.

\section{(Recebido para publicação em 9 de junho de 2014; aceito em 19 de março de 2015) (Received on June 9, 2014; accepted on March 19, 2015)}

$\mathrm{O}$ Brasil é um importante produtor de hortaliças, mas as perdas, principalmente pós-colheita, são igualmente altas. Estima-se que 35 a $45 \%$ destes produtos vegetais são perdidos ou desperdiçados, desde a classificação e seleção das olerícolas na propriedade rural até a sua utilização pelo consumidor final (Tofanelli et al., 2009). Um dos maiores problemas para a comercialização da alface é a sua alta perecibilidade em comparação com outras culturas. Idealmente, a qualidade da cabeça de alface deveria permanecer ao longo do tempo, facilitando sua comercialização e consumo (Schofield et al., 2005). A qualidade pós-colheita refere-se a vários aspectos, incluindo a aparência, qualidade sensorial e valor nutritivo.

A aparência é considerada um fator decisivo para a comercialização, podendo ser caracterizada por uma série de fatores como tamanho, formato, coloração e ausência/presença de defeitos perceptíveis. Vários fatores pré-colheita podem afetar a aparência do produto, incluindo a variabilidade genética e fatores biológicos, fisiológicos, agronômicos e ambientais (Hilton et al., 2009). Em alface, a aparência é comprometida principalmente pela desidratação (Aguero et al., 2008) e descoloração (Aguero et al., 2011; Bonasia et al., 2013) em função do tempo pós-colheita.

A desidratação de cabeças de alface em função do tempo de armazenamento, comumente é avaliada pela perda de água (Aguero et al., 2011). Já a descoloração da alface, tem sido eficientemente 
avaliada por meio da escala de notas (Aguero et al., 2008, 2011; Martínez-Sánchez et al., 2011; Atkinson et al., 2013a, 2013b).

Dentre as formas de estudar o declínio da qualidade dos vegetais em função do tempo após a colheita destaca-se o método gráfico. Neste método são ajustados modelos lineares ou não-lineares para cada tratamento. Posteriormente, são plotadas curvas a partir dos valores preditos pelos modelos de regressão. Assim, a comparação entre os tratamentos pode ser feita visualmente observando-se as curvas. Porém, esta estratégia pode ser comprometida, caso seja avaliado um elevado número de tratamentos, o que torna a representação gráfica de difícil visualização devido ao grande número de curvas. Neste contexto, o agrupamento de curvas pela similaridade dos coeficientes de regressão utilizando a análise multivariada torna-se uma alternativa viável e tem-se mostrado útil no estudo da produção de café (Cecon et al., 2008) e para o estudo do progresso de doenças em tomate (Fiorini et al., 2010; Azevedo et al., 2012).

Desta forma, objetivou-se selecionar cultivares de alface quanto à conservação pós-colheita por meio do agrupamento multivariado de curvas.

\section{MATERIAL E MÉTODOS}

O experimento foi conduzido de 15 de novembro de 2010 a 23 de março de 2011, no Setor de Olericultura, da Universidade Federal dos Vales do Jequitinhonha e Mucuri em Diamantina-MG (18 $12^{\circ} 01^{\prime}$ S, 4334'20"O, altitude de $1.387 \mathrm{~m}$ ). O solo é classificado como Neossolo Quartzarênico Órtico típico. No período em que o experimento foi conduzido, as temperaturas médias oscilaram entre 18,7 e $23,8^{\circ} \mathrm{C}$.

Foram avaliadas quinze cultivares de alface, sendo elas do tipo americana (Ironwood, 08Y472 (Perovana), Winslow e Teresa), lisa (Regina 500, Branca Boston, Quatro Estações, Vitória de Santo Antão, Atração e Lívia), crespa (Grand Rapids, Black Seed Simpson e Lollo Rossa), romana (Romana Balão) e repolhuda manteiga (Aurélia), sendo as sementes adquiridas em estabelecimentos comerciais da região.

A semeadura foi realizada em bandejas de poliestireno expandido de 128 células, utilizando-se o substrato comercial Plantmax Hortaliças, em casa de vegetação, sob sombrite com $50 \%$ de insolação. A área para o plantio foi preparada dois meses antes do transplantio das mudas por uma aração e uma gradagem, formando-se os canteiros manualmente, com enxada. As adubações de plantio, cobertura e os demais tratos culturais foram realizados de acordo com as recomendações para a cultura segundo Filgueira (2008).

O transplantio das mudas foi feito 30 dias após o semeio, em canteiros sob ambiente protegido, com aproximadamente $1,20 \mathrm{~m}$ de largura e $0,30 \mathrm{~m}$ de altura, utilizando-se espaçamento de $30 \times 30 \mathrm{~cm}$. O delineamento experimental utilizado foi de blocos ao caso, com quatro repetições, contendo doze plantas por parcela. A irrigação foi feita por gotejamento e micro-aspersão, seguindo o turno de rega conforme recomendação de Filgueira (2008), durante o período diurno.

Aos 45 dias após o transplantio das mudas, quando todas as cultivares apresentavam padrão comercial, foi realizada a colheita das cabeças de alface obtendo-se aleatoriamente três plantas em cada parcela. As plantas foram mantidas à sombra sobre bancadas em temperatura de $20 \pm 1^{\circ} \mathrm{C}$. Durante 72 horas, entre intervalos de 12 horas, foram avaliados o grau de descoloração e a perda acumulada de matéria fresca através da pesagem das cabeças em balança analítica, totalizando seis medições. O grau de descoloração foi obtido por 5 avaliadores previamente treinados, em escala de notas variando de 1 a 5 (1= ausência de descoloração;

Tabela 1. Média do coeficiente de determinação ajustado $\left(\mathrm{R}^{2}{ }_{\text {Ajust }}\right)$, do critério de informação Akaike (AIC) e porcentagem de convergência (Conv) obtidos a partir de modelos de regressão ajustados para cada parcela experimental no estudo da perda acumulada de matéria fresca e grau de descoloração em cabeças de alface (average adjusted coefficient of determination $\left(\mathrm{R}^{2}{ }_{\text {Ajust }}\right)$, Akaike information criterion (AIC) and percentage of convergence (Conv) from adjusted regression models obtained for each plot in the study of cumulative loss of fresh weight and degree of discoloration on lettuce heads). Diamantina, UFVJM, 2011.

\begin{tabular}{|c|c|c|c|c|c|c|}
\hline \multirow{2}{*}{ Modelos } & \multicolumn{3}{|c|}{$\begin{array}{l}\text { Perda acumulada de maté- } \\
\text { ria fresca }\end{array}$} & \multicolumn{3}{|c|}{ Grau de descoloração } \\
\hline & $\mathbf{R}_{\text {Ajust }}^{2}$ & AIC & $\begin{array}{l}\text { Conv } \\
(\%)\end{array}$ & $\mathbf{R}_{\text {Ajust }}^{2}$ & AIC & $\begin{array}{c}\text { Conv } \\
(\%)\end{array}$ \\
\hline Schnute & 0,456 & 36,852 & 98,333 & 0,488 & 24,208 & 100,000 \\
\hline Mitscherlich & 0,975 & 7,720 & 100,000 & 0,956 & 5,805 & 100,000 \\
\hline Richards & 0,940 & 16,415 & 93,333 & 0,959 & 4,723 & 100,000 \\
\hline Gompertz & 0,907 & 17,145 & 95,000 & 0,959 & 4,723 & 100,000 \\
\hline Logístico & 0,959 & 14,941 & 91,667 & 0,967 & 2,824 & 100,000 \\
\hline Meloun I & 0,969 & 7,746 & 100,000 & 0,956 & 5,805 & 100,000 \\
\hline Meloun II & 0,977 & 5,095 & 61,667 & 0,956 & 5,805 & 100,000 \\
\hline Brody & 0,969 & 7,720 & 100,000 & 0,908 & 10,802 & 100,000 \\
\hline von Bertalanffy & 0,976 & 9,626 & 91,667 & 0,959 & 3,014 & 100,000 \\
\hline Michaelis-Menten & 0,965 & 9,192 & 100,000 & 0,680 & 10,104 & 100,000 \\
\hline Exponencial & 0,900 & 21,974 & 100,000 & 0,653 & 18,910 & 100,000 \\
\hline Linear simples & 0,979 & 3,694 & - & 0,762 & 15,470 & - \\
\hline Linear quadrático & 0,964 & 9,694 & - & 0,929 & 6,913 & - \\
\hline
\end{tabular}


2= baixa descoloração; $3=$ descoloração moderada; 4= descoloração de moderada para severa; $5=$ descoloração severa). A perda acumulada de matéria fresca (PAMF) foi obtida através da equação:

PAMF $_{\mathrm{i}}(\%)=100\left(P_{0}-P_{i}\right) / P_{0}$

Onde $\mathrm{PAMF}_{\mathrm{i}}$ é a perda acumulada de matéria fresca no tempo i, $P_{0}$ é o peso da cabeça de alface no momento da colheita e $P_{i}$ é o peso da cabeça de alface no tempo $\mathrm{i}$.

A análise estatística foi feita em nível de média da parcela. Assim, para a perda de matéria fresca foi considerada a média das três plantas que constituíram as parcelas. Já para o grau de descoloração, foi considerada a média das notas atribuídas pelos cinco avaliadores nas três plantas que compuseram a parcela. Desta forma, em cada tempo de avaliação, houve quatro médias para cada cultivar, correspondente às quatro repetições. Para descrever o comportamento da perda acumulada de matéria fresca e grau de descoloração em função do tempo (horas após a colheita), adotou-se metodologia estatística similar à descrita por Azevedo et al. (2012), Cecon et al. (2008) e Fiorini et al. (2010), recorrendo-se ao uso de modelos de regressão e análise multivariada.

$\mathrm{Na}$ análise de regressão, além dos modelos linear simples $\left(y_{i}=\beta_{1}+\beta_{2} x_{i}\right.$ $\left.+\mathrm{e}_{\mathrm{i}}\right)$, linear quadrático $\left(\mathrm{y}_{\mathrm{i}}=\beta_{1}+\beta_{2} \mathrm{x}_{\mathrm{i}}+\right.$ $\left.\beta_{3} x_{i}^{2}+e_{i}\right)$ e exponencial $\left(y_{i}=\beta_{1} \exp \left(\beta_{3} x_{i}\right)\right.$ $+\mathrm{e}_{\mathrm{i}}$ ), foram testados também modelos mais complexos como o de Schnute, Mitscherlich, Richards, Gompertz, Logístico, Meloun I, Meloun II, Brody, von Bertalanffy e Michaelis-Menten, cujas funções são apresentadas por Silveira et al. (2011). Para todos os modelos, assumiu-se que $e_{i} \sim N\left(0, \sigma^{2}\right)$. Esses modelos foram ajustados para cada repetição de cada cultivar mediante o PROC MODEL do software SAS ${ }^{\circledR}$
(SAS, 1996) via método dos quadrados mínimos ordinários com algoritmo de Gauss-Newton. Foram utilizados como avaliadores da qualidade de ajuste o coeficiente de determinação ajustado $\left(\mathrm{R}^{2}{ }_{\mathrm{aj}}\right)$, o critério de informação de Akaike (AIC) e porcentagem de convergência (Conv).

Após a escolha do modelo, realizou-se uma análise de variância multivariada utilizando o PROC GLM (SAS, 1996) com a opção MANOVA, a fim de avaliar o efeito das cultivares sob as estimativas dos parâmetros do modelo. Foram extraídas as médias ajustadas destes coeficientes para cada tratamento (cultivar) por quadrados mínimos (LSMEANS). Estas médias foram padronizadas e utilizadas para o cálculo do quadrado da distância euclidiana. Posteriormente, foi feita a análise de agrupamento no PROC CLUSTER (SAS, 1996) pelo método do centróide.

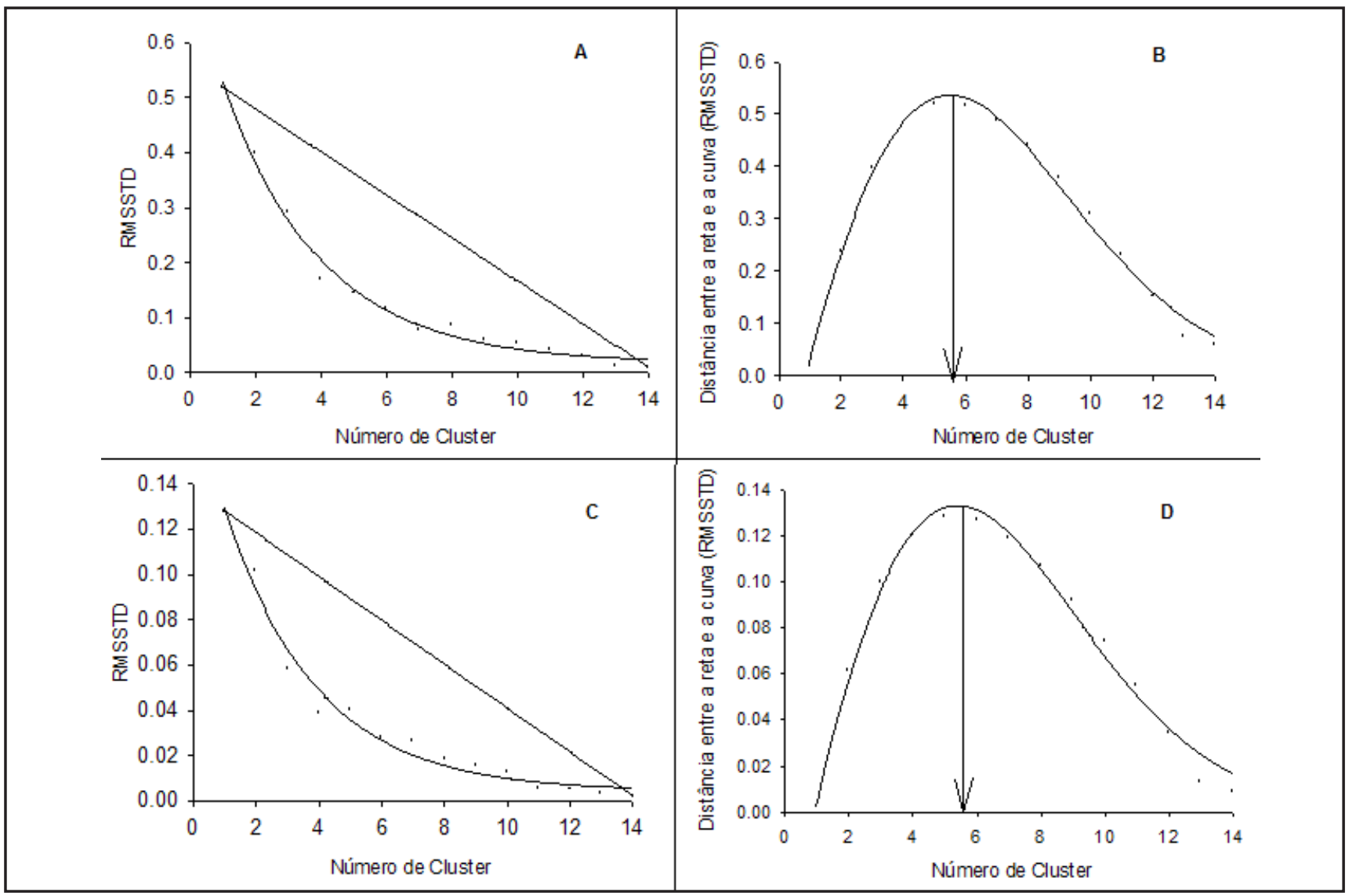

Figura 1. Comportamento da estatística desvio-padrão da raiz do quadrado médio (RMSSTD), e distância entre a reta e a curva estimada (RMSSTD) para verificação do número ótimo de grupos pelo método da máxima curvatura para a perda acumulada de matéria fresca (A e B) e grau de descoloração (C e D) (behavior of the statistical standard deviation of the root of mean square (RMSSTD), and distance between the line and the estimated curve (RMSSTD) to check the optimal number of cluster through the maximum curvature method for the cumulative loss of fresh weight (A and B) and degree of discoloration (C and D). Diamantina, UFVJM, 2011. 
A fim de identificar o número ótimo de cluster, foram obtidos os valores dos desvios-padrão da raiz do quadrado médio (RMSSTD), que permite a identificação do número ótimo de cluster por meio do ajuste do modelo de máxima curvatura. Segundo Cecon et al. (2008), esta análise tem a vantagem de possibilitar que o número de grupos não seja selecionado de forma subjetiva. Para visualizar o dendograma e verificar os grupos de cultivares utilizou-se o PROC TREE (SAS, 1996).

\section{RESULTADOS E DISCUSSÃO}

Os valores de $\mathrm{R}^{2}$ ajustado e AIC indicam que o modelo linear simples e logístico foram os que mais se adequaram ao estudo da perda acumulada de matéria fresca e grau de descoloração, respectivamente (Tabela 1). Resultados similares a estes, foram encontrados por Martínez-Romero et al. (2008) que, ao avaliar a perda de água em cabeças de alface em relação à vida de prateleira na temperatura de $20^{\circ} \mathrm{C}$, verificaram comportamento semelhante ao modelo linear simples. Para o grau de descoloração, Aguero et al. (2008) encontraram resultados parecidos em folhas externas, intermediárias e internas de cabeças de alface armazenadas em temperatura de 0 a $2^{\circ} \mathrm{C}$.

Associações entre o uso da escala de notas para a determinação da qualidade de alface e o tempo pós-colheita são encontradas em vários trabalhos na literatura (Schofield et al., 2005; Aguero et al., 2008; Ponce et al.,2011; Ansorena et al., 2012; Atkinson et al., 2013a). Comportamento similar ao logístico para o grau de descoloração também foi verificado por Atkinson et al. (2013a), que avaliaram a descoloração em alface minimamente processada por 13 dias sob a temperatura de $5^{\circ} \mathrm{C}$, utilizando também a escala de notas.

Foi observado na análise de variância multivariada pelos testes Wilks' lambda, Pillai's trace, Hotelling-lawley e Roy's root o efeito significativo das cultivares sobre o vetor dos parâmetros estudados. Isto indica a necessidade de mais de uma curva para explicar o progresso da desidratação e descoloração. Resultados que corroboram os obtidos foram encontrados por Azevedo et al. (2012), Cecon et al. (2008) e Fiorini et al. (2010).
Pelo método da máxima curvatura do RMSSTD verificou-se a possibilidade de redução das 15 curvas obtidas de cada uma das cultivares para seis grupos, tanto para a perda acumulada de matéria fresca quanto para o grau de descoloração (Figura 1), proporcionando a melhor visualização e interpretação dos dados. Utilizando esta mesma técnica, Cecon et al. (2008) explicaram o comportamento de 27 acessos de café com 8 grupos quanto à produtividade. Azevedo et al. (2012) explicaram o comportamento de 42 acessos de tomate dividindo-os em 6 grupos quanto a resistência à requeima e Fiorini et al. (2010) explicaram o comportamento de 27 acessos de tomate com 8 grupos quanto a resistência à requeima.

Para cada grupo formado (Figura 2) foi calculada, a partir das cultivares que os compuseram, a média e o desvio padrão das estimativas de $\hat{\beta}_{1}$ e $\hat{\beta}_{2}$ para a perda acumulada de matéria fresca e do $\hat{\beta}_{1}$ e $\hat{\beta}_{3}$ para o grau de descoloração (Tabela 2). Para o modelo linear simples o coeficiente $\hat{\beta}_{1}$ indica a média inicial, enquanto que o coeficiente angular da reta

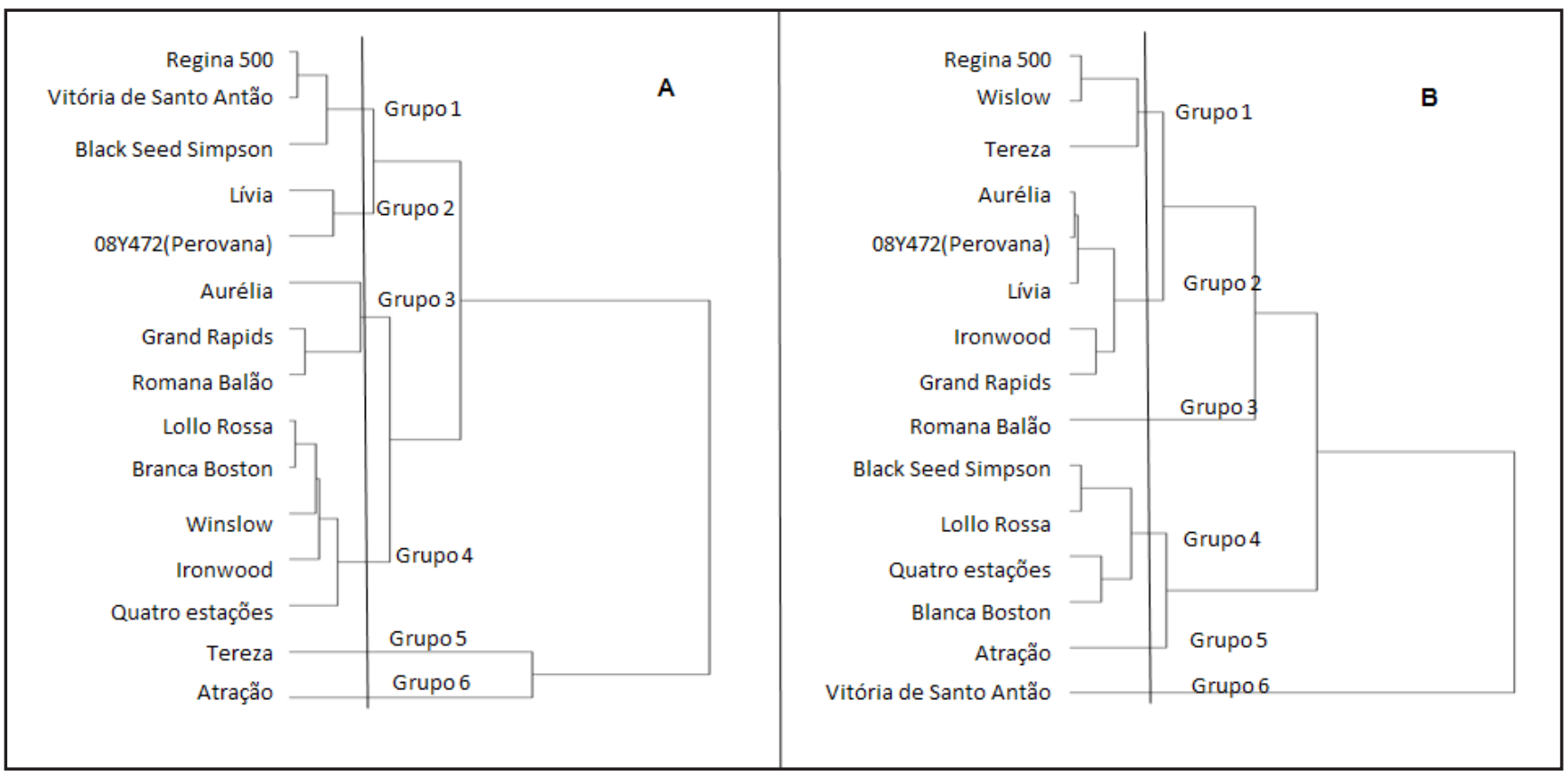

Figura 2. Dendrograma proveniente da análise de agrupamento para classificação das cultivares, considerando as estimativas dos parâmetros das curvas de perda acumulada de matéria fresca (A) e para o grau de descoloração (B) em cabeças de alface submetidas à temperatura ambiente (dendrogram from the cluster analysis to classify the cultivars, considering the estimates of the parameters of curves of accumulated loss of fresh matter (A) and for the degree of discoloration (B) on lettuce heads subjected to room temperature). Diamantina, UFVJM, 2011. 
Tabela 2. Valores médios dos coeficientes de regressão e seus respectivos desvios padrões obtidos a partir das cultivares que constituíram cada agrupamento, baseando-se no modelo linear simples para a perda acumulada de matéria fresca e logístico para o grau de descoloração (average values of regression coefficients and respective standard deviation obtained from cultivars that constituted each cluster, based on the linear polynomial model for the cumulative loss of fresh mater and logistics for the degree of discoloration). Diamantina, UFVJM, 2011.

\begin{tabular}{|c|c|c|c|c|c|c|c|c|}
\hline \multirow{2}{*}{ Grupo } & \multicolumn{4}{|c|}{$\begin{array}{c}\text { Perda acumulada de matéria } \\
\text { fresca }\end{array}$} & \multicolumn{4}{|c|}{ Grau de descoloração } \\
\hline & $\hat{\beta}_{1}$ & $\mathbf{D P}\left(\hat{\beta}_{1}\right)$ & $\hat{\beta}_{2}$ & $\mathbf{D P}\left(\hat{\beta}_{2}\right)$ & $\hat{\beta}_{1}$ & $\mathbf{D P}\left(\hat{\beta}_{1}\right)$ & $\hat{\beta}_{2}$ & $\mathbf{D P}\left(\hat{\beta}_{2}\right)$ \\
\hline 1 & 0,362 & 0,087 & 0,357 & 0,005 & 1,885 & 0,040 & 0,051 & 0,001 \\
\hline 2 & 0,702 & 0,122 & 0,358 & 0,016 & 1,791 & 0,025 & 0,049 & 0,006 \\
\hline 3 & $-0,444$ & 0,147 & 0,359 & 0,080 & 1,443 & - & 0,058 & - \\
\hline 4 & $-0,043$ & 0,101 & 0,420 & 0,041 & 2,037 & 0,038 & 0,051 & 0,001 \\
\hline 5 & 1,289 & - & 0,342 & - & 2,135 & - & 0,050 & - \\
\hline 6 & 2,256 & - & 0,496 & - & 1,638 & - & 0,052 & - \\
\hline
\end{tabular}

$\left(\hat{\beta}_{2}\right)$ indica a taxa de progresso. Já para os modelos não-lineares analisados, segundo Silveira et al. (2011), o parâmetro $\hat{\beta}_{1}$ representa a média final, o parâmetro $\hat{\beta}_{2}$ não apresenta interpretação prática e de inflexão fixo, ou não o possuem.

Para a perda acumulada de matéria fresca, verificou-se melhor comportamento para o grupo 3, formado pelas cultivares Aurélia, Grand Rapids e Romana Balão, com menores valores de $\hat{\beta}_{1}(-0,444)$, refletindo menor perda de matéria fresca nos momentos iniciais de avaliação e menor $\hat{\beta}_{1}$ indicando uma menor taxa de perda de matéria fresca. $\mathrm{O}$ mesmo é verificado na Figura 3a, onde o grupo 3 apresentou melhor comportamento, devido ao fato de aproximar-se mais do eixo X. Já o grupo 6, formado pela cultivar Atração apresentou os piores resultados, com altos valores de $\hat{\beta}_{1}(2,256)$ e $\hat{\beta}_{2}(0,496)$.

Quanto ao grau de descoloração, o grupo 6 formado pela cultivar Vitória de Santo Antão apresentou melhor resultado baseando-se nos valores de $\hat{\beta}_{1}(1,638), \hat{\beta}_{2}(0052)$ e na Figura $3 b$, enquanto que o grupo 5 formado pela cultivar 'Atração' apresentou os piores resultados para o grau de descoloração (Figura 3b), apresentando maior valor

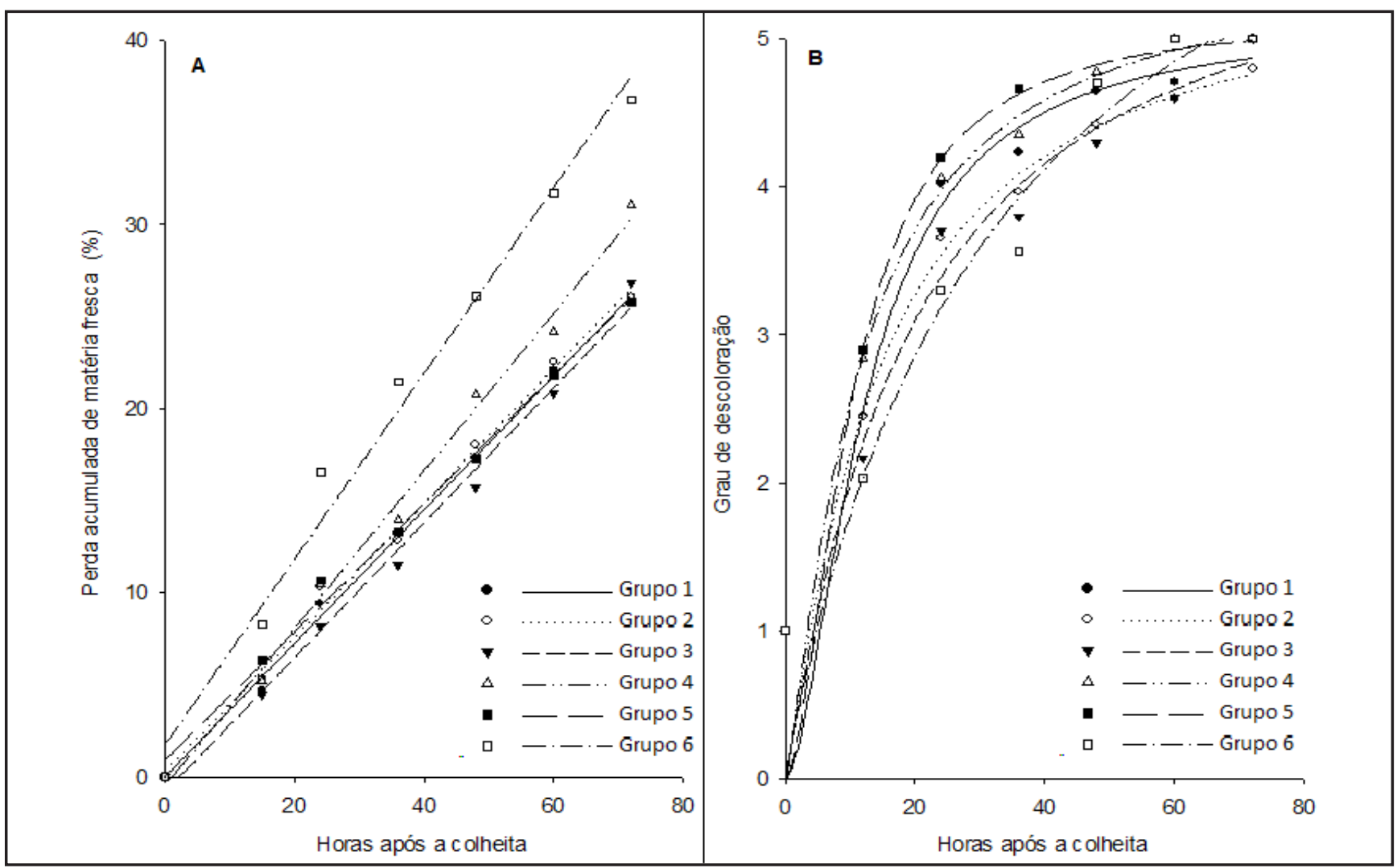

Figura 3. Curvas de perda acumulada de matéria fresca (A) e grau de descoloração (B) em cabeças de alface para cada grupo obtido pela análise multivariada (curves of cumulative loss of fresh mater (A) and degree of discoloration (B) on lettuce heads for each group obtained by multivariate analysis). Diamantina, UFVJM, 2011. 
de $\hat{\beta}_{1}$ (Tabela 2).

Além das cultivares Romana Balão e Vitória de Santo Antão fazerem parte daquelas com menor perda acumulada de matéria fresca e menor grau de descoloração, apresentaram também as maiores produtividades encontradas por Azevedo et al. (2013) avaliando 15 cultivares de alface. Assim, conclui-se que as cultivares 'Romana Balão' e 'Vitória de Santo Antão’ são as mais indicadas para a produção, comercialização e como genitores em programa de melhoramento com a cultura da alface.

\section{AGRADECIMENTOS}

À FAPEMIG, ao CNPq e à CAPES pela concessão de bolsas de estudos e recursos financeiros para o desenvolvimento do projeto.

\section{REFERÊNCIAS}

AGÜERO MV; BARG MV; YOMMI A; CAMELO A; ROURA SI. 2008. Postharvest changes in water status and chlorophyll content of lettuce (Lactuca sativa) and their relationship with overall visual quality. Journal of Food Science 73: 47-55.

AGÜERO MV; PONCE AG; MOREIRA MR; ROURA SI. 2011. Lettuce quality loss under conditions that favor the wilting phenomenon. Postharvest Biology and Technology 59: 124-131.

ANSORENA MR; AGÜERO MV; GOÑI MG; ROURA S; PONCE A; MOREIRA MR; DI SCALA K. 2012. Assessment of lettuce quality during storage at low relative humidity using Global Stability Index methodology. Ciência e Tecnologia de Alimentos 32: 366-373.

ATKINSON LD; HILTON HW; PINK DAC. 2013a. A study of variation in the tendency for postharvest discoloration in a lettuce (Lactuca sativa) diversity set. International Journal of Food Science and Technology 48: 801-807.

ATKINSON LD; MCHALE LK; TRUCO MJ; HILTON HW; LYNN J; SCHUT JW; MICHELMORE RW; HAND P; PINK DAC. 2013b. An intra-specific linkage map of lettuce (Lactuca sativa) and genetic analysis of postharvest discoloration traits. Theor Appl Genet 126: 2737-2752.

AZEVEDO AM; ANDRADE JÚNIOR VC; OLIVEIRA CM; FERNANDES JSC; PEDROSA CE; DORNAS MFS; CASTRO BMC. 2013. Seleção de genótipos de alface para cultivo protegido: divergência genética e importância de caracteres. Horticultura Brasileira 31: 260-265.

AZEVEDO CF; SILVA FF; RIBEIRO NB; SILVA DJH; CECON PR; BARIL LD; PINHEIRO VR. 2012. Classificação multivariada de curvas de progresso da requeima do tomateiro entre acessos do Banco de Germoplasma de Hortaliças da UFV. Ciência Rural 42: 414-417.

BONASIA A; CONVERSA G; LAZZIZERA C; ELIA A. 2013. Pre-harvest nitrogen and azoxystrobin application enhances postharvest shelf-life in butterhead lettuce. Postharvest Biology and Technology 85: 67-76.

CECON PR; SILVA FF; FERREIRAA; FERRÃO RG; CARNEIRO APS; DETMANN E; FARIA PN; MORAIS TSS. 2008. Análise de medidas repetidas na avaliação de clones de café 'Conilon'. Pesquisa Agropecuária Brasileira 43: 1171-1176.

FILGUEIRA FAR. 2008. Novo manual de olericultura: Agrotecnologia moderna na produção e comercialização de hortaliças. Viçosa: UFV. 402p.

FIORINI CVA; SILVA DJH; SILVA FF; MIZUBUTI ESG; ALVES DP; CARDOSO TS. 2010. Agrupamento de curvas de progresso de requeima, em tomateiro originado de cruzamento interespecífico. Pesquisa Agropecuária Brasileira 45: 1095-1101.

HILTON HW; CLIFFORD SC; WURR DCE; BURTON KS. 2009. The influence of agronomic factors on the visual quality of fieldgrown, minimally-processed lettuce. Journal of Horticultural Science \& Biotechnology 84: 193-198.

MARTÍNEZ-ROMERO D; SERRANO M; BAILÉN G; GUILLÉN F; ZAPATA PJ; VALVERDE JM; CASTILLO S; FUENTES M; VALERO D. 2008. The use of a natural fungicide as an alternative to pre harvest synthetic fungicide treatments to control lettuce deterioration during postharvest storage. Postharvest Biology and Technology 47: 54-60.

MARTÍNEZ-SÁNCHEZ A; TUDELA JA; LUNA C; ALLENDE A; GIL MI. 2011. Low oxygen levels and light exposure affect quality of fresh-cut Romaine lettuce. Postharvest Biology and Technology 59: 34-42.

PONCE A; ROURA SI; MOREIRA MR. 2011. Essential oils as biopreservatives: different methods for the technological application in lettuce leaves. Journal of Food Science 76: 34-40.

SAS. 1996. User's guide, version 6.11. Cary: SAS Institute. 842p.

SCHOFIELD RA; DEELL JR; MURR DP; JENNI S. 2005. Determining the storage potential of iceberg lettuce with chlorophyll fluorescence. Postharvest Biology and Technology 38: 43-56.

SILVEIRA FG; SILVA FF; CARNEIRO PS; MALHADO CHM; MUNIZ JA. 2011. Análise de agrupamento na seleção de modelos de regressão não-lineares para curvas de crescimento de ovinos cruzados. Ciência Rural 41: 692- 698

TOFANELLI MBD; FERNANDES MS; CARRIJO NS; MARTINS FILHO OB. 2009. Levantamento de perdas em hortaliças frescas na rede varejista de Mineiros. Horticultura Brasileira 27: 116-120. 